\title{
Effects of cannabinoid receptor type 2 on endogenous myocardial regeneration by activating cardiac progenitor cells in mouse infarcted heart
}

\author{
WANG YaBin ${ }^{1 \dagger}$, MA Sai $^{1 \dagger}$, WANG Qiang ${ }^{2}$, HU WenXing ${ }^{1}$, WANG DongJuan ${ }^{1}$, LI XiuJuan ${ }^{1}$, \\ SU Tao ${ }^{1}$, QIN Xing ${ }^{1}$, ZHANG XiaoTian ${ }^{1}$, MA Ke ${ }^{1}$, CHEN JiangWei ${ }^{1}$, XIONG LiZe $^{2}$ \& \\ CAO Feng ${ }^{1,3 *}$
}

${ }^{1}$ Department of Cardiology, Xijing Hospital, Fourth Military Medical University, Xi'an 710032, China; ${ }^{2}$ Department of Anesthesiology, Xijing Hospital, Fourth Military Medical University, Xi'an 710032, China; ${ }^{3}$ Department of Cardiology, PLA General Hospital, Beijing 100853, China

Received August 7, 2013; accepted September 20, 2013; published online January 14, 2014

\begin{abstract}
Cannabinoid receptor type $2\left(\mathrm{CB}_{2}\right)$ activation is recently reported to promote proliferation of some types of resident stem cells (e.g., hematopoietic stem/progenitor cell or neural progenitor cell). Resident cardiac progenitor cell (CPC) activation and proliferation are crucial for endogenous cardiac regeneration and cardiac repair after myocardial infarction (MI). This study aims to explore the role and possible mechanisms of $\mathrm{CB}_{2}$ receptor activation in enhancing myocardial repair. Our results revealed that $\mathrm{CB}_{2}$ receptor agonist AM1241 can significantly increase CPCs by c-kit and Runx1 staining in ischemic myocardium as well as improve cardiomyocyte proliferation. AM1241 also decreased serum levels of MDA, TNF- $\alpha$ and IL-6 after MI. In addition, AM1241 can ameliorate left ventricular ejection fraction and fractional shortening, and reduce fibrosis. Moreover, AM1241 treatment markedly increased p-Akt and HO-1 expression, and promoted Nrf-2 nuclear translocation. However, PI3K inhibitor wortmannin eliminated these cardioprotective roles of AM1241. In conclusion, AM1241 could induce myocardial regeneration and improve cardiac function, which might be associated with PI3K/Akt/Nrf2 signaling pathway activation. Our findings may provide a promising strategy for cardiac endogenous regeneration after MI.
\end{abstract}

$\mathrm{CB}_{2}$ receptor, cardiac progenitor cells, endogenous cardiac regeneration

Citation: Wang YB, Ma S, Wang Q, Hu WX, Wang DJ, Li XJ, Su T, Qin X, Zhang XT, Ma K, Chen JW, Xiong LZ, Cao F. Effects of cannabinoid receptor type 2 on endogenous myocardial regeneration by activating cardiac progenitor cells in mouse infarcted heart. Sci China Life Sci, 2014, 57: 201-208, doi: $10.1007 / \mathrm{s} 11427-013-4604-\mathrm{z}$

Myocardial infarction (MI) and its complications are the leading causes of mortality and morbidity worldwide. Revascularization and medication therapy are unable to reconstitute the necrotic myocardium and improve cardiac function. In recent years, stem-cell therapy emerged as a promising strategy for patients after MI [1]. Nevertheless, clinical applications of exogenous stem-cell therapy are seriously

$\dagger$ Contributed equally to this work

*Corresponding author (email: wind8828@gmail.com) hindered because it is time-consuming and costly to isolate and expand stem cells, as well as due to poor retention and survival capacity after engraftment [2]. Thus, in situ activation, proliferation and differentiation of endogenous stem cells attract significant interest. Under normal conditions, multipotent cardiac progenitor cells (CPCs) that are present in the mammalian heart maintain homeostasis. Once they receive some stimulation, such as pharmacological administration, growth-factor intervention or microRNA regulation, these CPCs can be activated in situ to differentiate into car- 
diomyocytes that promote myocardial regeneration [3-5].

The endocannabinoid system, especially the cannabinoid receptor-type 2 (CB2 receptor), has been recently reported to be involved in cardioprotective effects on MI injury [6]. Nevertheless, precise mechanisms have not been elucidated clearly. Some studies suggested that cardioprotective roles of the CB2 receptor could be partially attributed to anti-inflammation and anti-oxidative stress of the infarcted myocardium [7]. Interestingly, Palazuelos et al. [8] revealed that CB2-selective agonists promoted neural progenitor cell proliferation via activating the PI3K/Akt/mTORC1 signaling pathway. Moreover, Jiang et al. [9] reported that cannabinoid receptor 2 and its agonists modulated hematopoietic stem and progenitor cell mobilization and function. However, whether CB2 receptor sensitization could activate CPCs in situ and promote myocardial regeneration is still unknown.

In the present research, we hypothesized that $\mathrm{CB} 2$ selective agonists (AM1241) could activate CPCs in situ, promoting endogenous regeneration of myocardium and cardiac repair after MI, and preliminarily elucidated the potential mechanism.

\section{Materials and methods}

\subsection{Mice model of myocardial infarction}

C57BL/6 male mice (8-week-old, weighing 20-25 g) were randomly allocated into the following groups ( $n=20$ each): (i) Sham group (Sham), (ii) MI+PBS group (MI), (iii) MI+CB2 agonist AM1241 group (MI+AM), (iv) MI+CB2 agonist AM1241+Wortmannin group (MI+AM+W).

Mice myocardial infarction was induced by ligation of the left anterior descending (LAD) artery [10]. In brief, mice were anesthetized with persistent inhaled $2 \%$ isoflurane during the operation. A left thoracotomy was performed and the pericardium was opened. The LAD was permanently ligated with a 6-0 suture just at the level of the left atrium. The ligation was deemed successful when the anterior wall of the LV turned pale. For the sham-operated mice, open thoracotomy was performed without suturing of the LAD. The chest cavity was closed in layers with 4-0 suture. In the MI+AM group, AM1241 (20 mg kg ${ }^{-1} \mathrm{~d}^{-1}$; Selleck, USA) was intraperitoneally injected into mice for seven consecutive days after MI. In the MI+AM+W group, Wortmannin (15 $\mu \mathrm{g} \mathrm{kg}^{-1} \mathrm{~d}^{-1}$; Sigma, USA) was injected via tail vein for seven consecutive days after MI. All animal procedures were conducted in conformity with the National Institutes of Health Guide for the Care and Use of Laboratory Animals and all experiments were performed in accordance with the Helsinki Declaration.

\subsection{Echocardiography}

Echocardiography studies were performed with Vevo®
2100 ultrasound system (VisualSonics, CA) using a $30 \mathrm{MHz}$ linear-array ultrasound transducer on baseline (day-1) and POD 3, 14 and 28 by a blind investigator. The mice were anesthetized with inhaled $2 \%$ isoflurane and put in a supine position. Both two-dimensional and M-mode images were recorded. The left-ventricular end-systolic volume (LVESV) and left-ventricular end-diastolic volume (LVEDV) were measured to calculate left-ventricular ejection fraction (LVEF) and fractional shortening (FS).

\subsection{Masson's trichrome staining}

The mice were sacrificed and hearts were harvested for histological staining at POD 28. Separate sets of paraffin-embedded tissue sections were stained using Masson's trichrome, resulting in fibrotic (collagen-enriched) areas that appeared blue while cellular elements appeared red. For 10 randomly microscopic fields (200× magnification) of each LV, Masson's trichrome-stained myocardial sections were imaged, and the collagen area was calculated as a percentage of the total LV myocardial area. This served as an estimate of the progression of fibrosis. This calculation was performed by an observer blinded to the treatment groups.

\subsection{Immunofluorescence}

CPC and cardiomyocyte proliferation was detected with immunostaining for CPC surface marker c-kit, Runx 1 and proliferating cell nuclear marker ki67, respectively. Firstly, mice were anesthetized seven days after LAD ligation. The heart tissue was fixed in 4\% paraformaldehyde buffer and $5 \mu \mathrm{m}$ thick sections were prepared. Then, tissue sections were stained with primary anti-c-kit $(1: 200$, Abcam), antiRunx1 (1:200, Abcam) and anti-ki67 (1:50, Abcam) antibodies, respectively. 4,6-Diamidino-2-phenylindole (DAPI) was used to label cell nuclei. Immunofluorescence detection was performed with a laser-scanning confocal microscope (Olympus FV10i, Japan). The cells that were positive for c-kit, Runx 1 and ki67 were counted using the Image-ProPlus software (version 6.0; National Institutes of Health) in at least five high-power fields (HPF, 200x) in the infarct and peri-infarct regions, which were randomly selected and counted in at least three sections from each animal $(n=10$ per group).

\subsection{ELISA assay}

On POD 3, the blood sample (2 mL) was collected from the right common carotid artery and placed into Eppendorf tubes. After leaving at room temperature for $4 \mathrm{~h}$, the samples were centrifuged for $20 \mathrm{~min}\left(3000 \times g, 4^{\circ} \mathrm{C}\right)$. Aliquots of the supernatant were removed and stored at $-80^{\circ} \mathrm{C}$ before assay. According to the manufacturer's instructions, serum levels of Malondialdehyde (MDA), TNF- $\alpha$ and IL-6 were measured by colorimetric analysis using a spectrophotome- 
ter at a wavelength of $450 \mathrm{~nm}$ with the relevant detection kits (BIKW, China).

\subsection{Western blot analysis}

Western blot samples were extracted from myocardium on POD 7. The heart tissue was then lysed in an ice-cold RIPA buffer containing $1 \%$ protease inhibitor cocktail (Sigma, USA). The sample was centrifuged at $12000 \times g$ for $20 \mathrm{~min}$ at $4^{\circ} \mathrm{C}$. The supernatant was transferred, aliquoted and stored at $-80^{\circ} \mathrm{C}$. After protein concentration measurements by the modified Bradford assay (Bio-Rad Laboratories, Hercules, USA), the proteins were separated by SDS-PAGE electrophoresis, transferred to nitrocellulose membranes and probed with primary antibodies against HO-1 (1:1000, Abcam, USA), Nrf2 (1:1000, Abcam, USA), p-Akt (1:1000, Abcam, USA) overnight at $4{ }^{\circ} \mathrm{C}$ followed by incubation with the corresponding secondary antibodies at room temperature for $1 \mathrm{~h}$. The signal was detected using the ECL-Plus reagent (GE Healthcare, USA).

\subsection{Statistical analysis}

All statistical analyses were performed by SPSS software (Version 15.0, SPSS Inc., USA). All values are expressed as means \pm standard deviation (SD). Statistical differences between different groups were analyzed using one-way ANOVA, two-tailed unpaired $t$-test or chi-square test. Statistical significance was set at $P<0.05$.

\section{Results}

\subsection{AM1241 treatment improved cardiac function af- ter MI}

Echocardiogram was performed to evaluate cardiac function at baseline and on post-operative days (POD). The results revealed that there was no significant difference among all groups at baseline $(P>0.05)$. However, LVEF and FS decreased markedly in all groups on POD 3. AM1241 treatment improved LVEF and FS significantly as compared with that of the MI group on POD 14 (EF: $43.12 \% \pm 0.32 \%$ vs. $37.45 \% \pm 0.21 \%, P<0.05$; FS: $26.91 \% \pm 0.13 \%$ vs. $23.57 \%$ $\pm 0.22 \%, P<0.05)$ and POD $28(\mathrm{EF}: 47.63 \% \pm 0.37 \%$ vs. $39.82 \% \pm 0.48 \%, P<0.05$; FS: $28.36 \% \pm 0.21 \%$ vs. $24.01 \% \pm$ $0.25 \%, P<0.05)$ respectively. Wortmannin reversed the trend induced by AM1241 $(P<0.05)$ (Figure 1$)$.

\subsection{AM1241 treatment decreased fibrosis after MI}

To detect myocardial fibrosis in the infarcted heart with or without the AM1241 treatment, Masson's trichrome staining was carried out. Severe fibrosis was observed in the MI group, whereas the AM1241 intervention obviously reduced fibrosis as compared with that in the MI group $(29.4 \% \pm$ $1.03 \%$ vs. $41.36 \% \pm 1.60 \%, P<0.05)$. However, Wortmannin eliminated fibrosis-area decrease caused by the AM1241 administration $(39.62 \% \pm 1.04 \%$ vs. $29.4 \% \pm 1.03 \%, P<0.05)$ (Figure 2A and B).

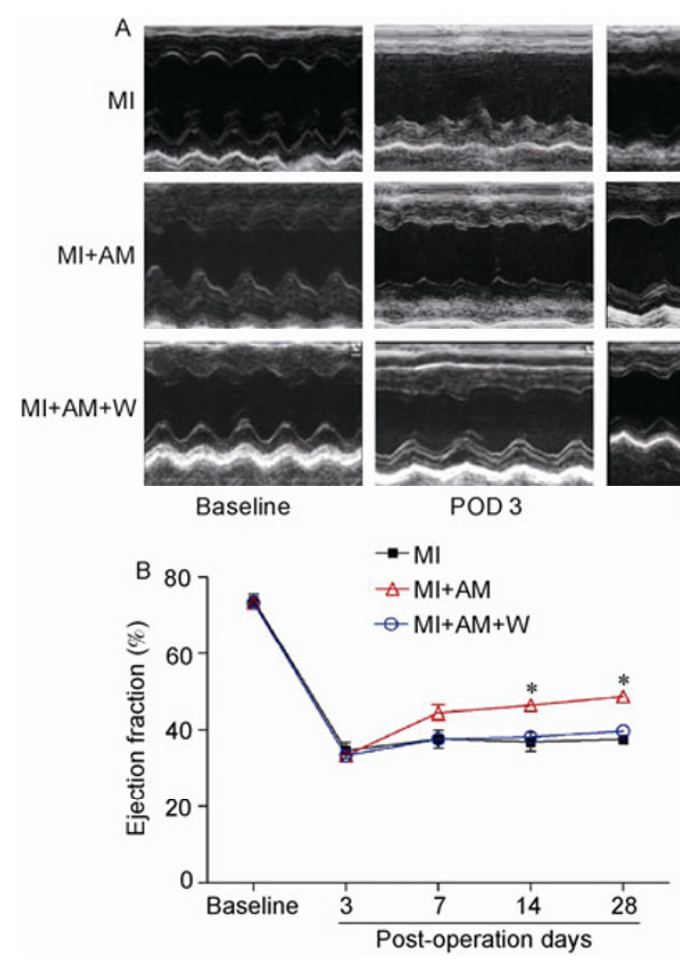

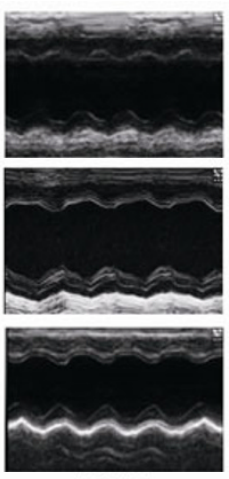

POD 7$$
\text { C }
$$

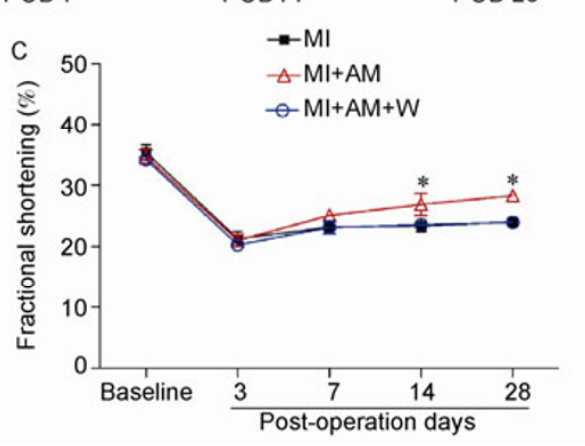

Figure 1 AM1241 improved cardiac function after myocardial infarction. A, The dynamic changes of cardiac function visualized by in vivo echocardiography. B and C, Quantification analysis of ejection fraction (EF) and fractional shortening (FS). ${ }^{*}, P<0.05$ vs. MI group. 

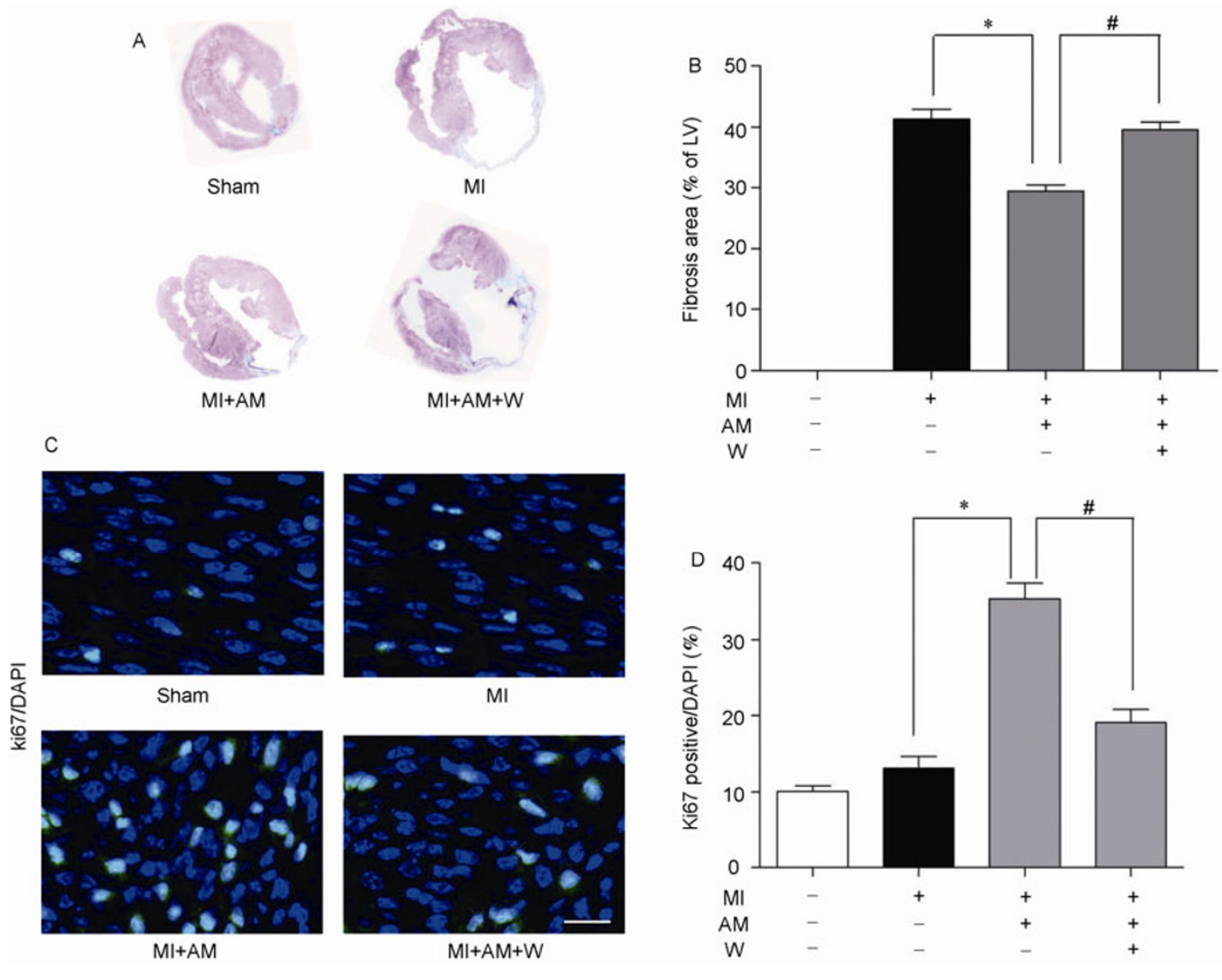

Figure 2 AM1241 increased ki67+ positive cells and decreased cardiac fibrosis after MI. A, Masson's trichrome-stained myocardial sections from a subgroup of animals at POD 28. B, Comparison of fibrosis areas in all groups. *, $P<0.05 \mathrm{vs.} \mathrm{MI} \mathrm{group;} \mathrm{\# ,} P<0.05$ vs. MI+AM group. C, Immunohistochemistry of Ki67 (green), and DAPI (blue) staining in adjacent infarction areas. D, Comparison of ki67 positive cells in all groups. *, $P<0.05$ vs. MI group; \#, $P<0.05$ vs. MI+AM group. Scale bar, $20 \mu \mathrm{m}$.

2.3 AM1241 treatment increased the number of c-kit ${ }^{+}$ and Runx $1^{+}$cells and cardiomyocyte proliferation in the myocardium after MI

c-kit and Runx1 are typical markers of cardiac progenitor/stem cells. Thus, c-kit and Runx1 immunofluorescence staining was performed to evaluate the number of progenitor/stem cells in the infarcted myocardium after the AM1241 treatment. The results revealed that the expression of c-kit and Runx1 was elevated significantly in the MI+AM group as compared with that in the MI group on POD 7 (c-kit: $8.67 \% \pm 0.72 \%$ vs. $4.27 \% \pm 0.56 \%, P<0.05$; Runx1: $9.92 \% \pm 0.77 \%$ vs. $5.54 \% \pm 0.68 \%, P<0.05$ ). However, Wortmannin eliminated AM1241-induced c-kit and Runx1 up-regulation (c-kit: $4.47 \% \pm 0.38 \%$ vs. $8.67 \% \pm 0.72 \%$, $P<0.05$; Runx1: $6.30 \% \pm 0.55 \%$ vs. $9.92 \% \pm 0.77 \%, P<0.05$ ) (Figure 3).

To detect proliferating cardiomyocytes, heart sections were immunostained with ki67. Interestingly, AM1241 further raised the number of ki67 $7^{+}$cardiomyocytes in comparison with the MI group on POD $7(35.32 \% \pm 2.03 \%$ vs. $13.14 \% \pm 1.48 \%, P<0.05$ ). Wortmannin reversed the increase of ki67 $7^{+}$cardiomyocytes in the infarcted heart caused by the AM1241 administration $(19.08 \% \pm 1.72 \%$ vs. $35.32 \% \pm$ $2.03 \%, P<0.05$ ) (Figure $2 \mathrm{C}$ and $\mathrm{D}$ ).

\subsection{AM1241 treatment decreased MDA, TNF- $\alpha$ and IL-6 levels in the infarcted myocardium}

MDA caused by oxidative damage serves as a biomarker of cardiac oxidative stress injury. Thus, we detected serum concentration of MDA to reflect oxidative stress in myocardium with or without the AM1241 administration. The data showed that MI significantly increased MDA levels $\left(9.34 \pm 0.59 \mathrm{nmol} \mathrm{mL}^{-1}\right.$ vs. $\left.4.40 \pm 0.71 \mathrm{nmol} \mathrm{mL}^{-1}, P<0.05\right)$ in comparison with the sham group. Moreover, the AM1241 administration significantly decreased MDA levels as compared with those in the MI group $\left(6.57 \pm 0.42 \mathrm{nmol} \mathrm{mL}^{-1}\right.$ vs. $9.34 \pm 0.59 \mathrm{nmol} \mathrm{mL}^{-1}, P<0.05$ ) (Figure $4 \mathrm{~A}$ ).

Next, we explored the MI-induced inflammation by assessing the levels of inflammatory cytokines TNF- $\alpha$ and IL-6. The ELISA analysis revealed that MI led to significant increases in TNF- $\alpha$ and IL-6, as compared with those in the sham group (TNF- $\alpha$ : $19.10 \pm 0.85 \mathrm{pg} \mathrm{mL}^{-1}$ vs. $10.34 \pm 1.15 \mathrm{pg}$ $\mathrm{mL}^{-1}, P<0.05$; IL-6: $16.53 \pm 0.63 \mathrm{pg} \mathrm{mL}^{-1}$ vs. $8.33 \pm 0.60 \mathrm{pg}$ 

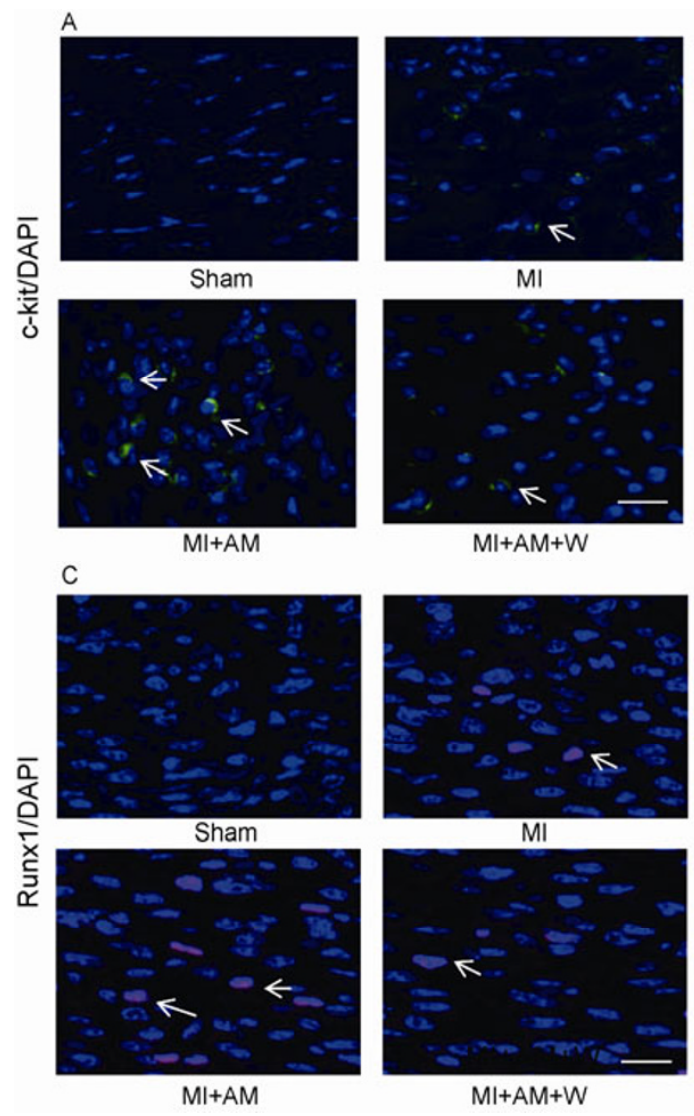

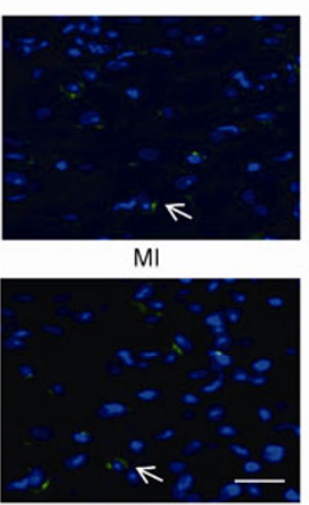

$\mathrm{Ml}+\mathrm{AM}+\mathrm{W}$
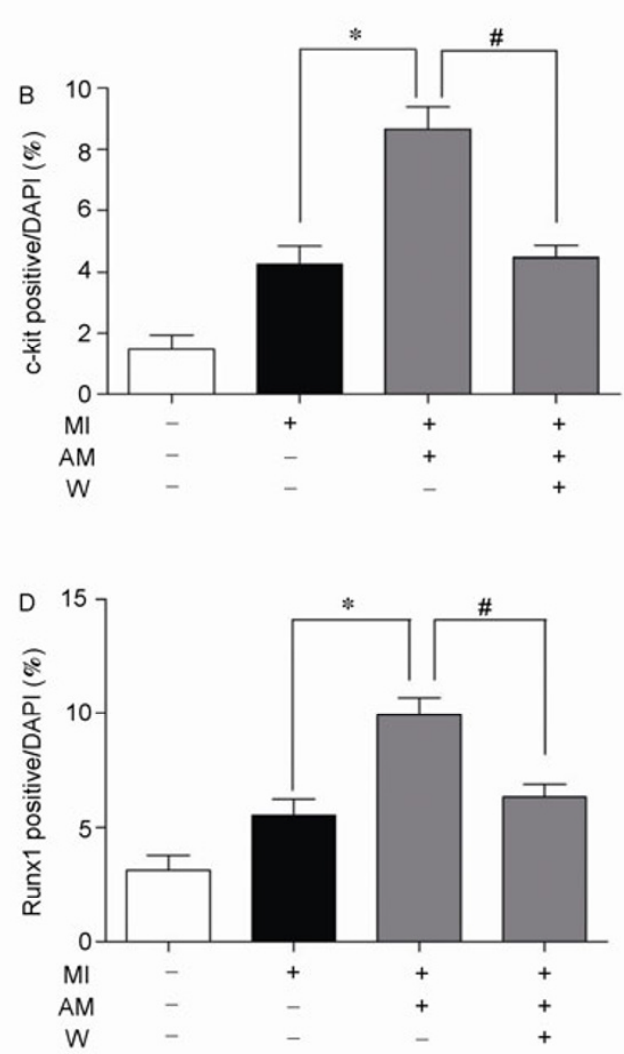

Figure 3 Effects of AM1241 on cardiac progenitor cell activation in vivo after myocardial infarction. A, Immunohistochemistry of c-kit (Green) and DAPI (Blue) staining in adjacent infarction areas. B, Comparison of c-kit positive cells in all groups. *, $P<0.05$ vs. the MI group; \#, $P<0.05$ vs. MI+AM group. C, Immunohistochemistry of Runx1 (red) and DAPI (blue) staining in adjacent infarction areas. $\mathrm{D}$, Comparison of Runx 1 positive cells in all groups. $*, P<0.05$ vs. MI group; \#, $P<0.05$ vs. MI+AM group. Scale bar, $20 \mu \mathrm{m}$.

$\left.\mathrm{mL}^{-1}, P<0.05\right)$. However, AM1241 markedly decreased the serum levels of TNF- $\alpha$ and IL- 6 induced by MI (TNF- $\alpha$ : $14.05 \pm 1.12 \mathrm{pg} \mathrm{mL}^{-1}$ vs. $19.10 \pm 0.85 \mathrm{pg} \mathrm{mL}^{-1}, P<0.05$; IL-6: $13.21 \pm 0.92 \mathrm{pg} \mathrm{mL} \mathrm{m}^{-1}$ vs. $\left.16.53 \pm 0.63 \mathrm{pg} \mathrm{mL}^{-1}, P<0.05\right)$. Wortmannin could reverse this trend (Figure $4 \mathrm{~B}$ and $\mathrm{C}$ ).

\subsection{AM1241 treatment increased Akt phosphorylation, Nrf2 nuclear translocation and HO-1 expression}

The PI3K/Akt/Nrf-2 signaling pathway was recently found to be related to resistance to oxidative stress and scavenging oxygen-free radicals. In our study, we intended to investigate whether AM1241 plays anti-oxidant and antiinflammation roles through modulating the PI3K/Akt/ Nrf-2 signaling pathway. The Western blot results showed that the p-Akt and HO-1 expression was significantly elevated in the AM1241 group as compared with that in the MI group $(P<0.05)$. Meanwhile, the Nrf-2 levels in the nuclear protein were also markedly increased in the AM1241 group as compared to that in the MI group $(P<0.05)$. These data indicated that AM1241 increased Akt phosphorylation, Nrf2 nuclear translocation and HO-1 expression in the infarcted myocardium, whereas Wortmannin blocked these effects of
AM1241 $(P<0.05)$ (Figure 5).

\section{Discussion}

In the present study, we found that selective CB2 agonist AM1241 could improve cardiac function and myocardial fibrosis after MI, which was related to activation of resident CPCs and promotion of endogenous cardiac regeneration. Furthermore, our study revealed that AM1241 ameliorated adverse oxidative stress and inflammation milieu in infarcted heart through up-regulating the PI3K/Akt/Nrf-2 signal pathway.

The endocannabinoid system comprises two major membrane receptors (CB1 and $\mathrm{CB} 2$ ), the endogenous ligands and specific enzymes for their degradation and inactivation. The CB1 receptor is mainly located in the central nervous system, while the CB2 receptor is predominantly expressed in peripheral tissue and cells, such as lymphocytes, neutrophils and cardiomyocytes [8,11-13]. In addition to immunomodulatory and anti-inflammation properties, the $\mathrm{CB} 2$ receptor activation has been recently shown to promote myocyte survival and to attenuate ischemia/ reperfusion injury in cardiac tissue [14]. Our echocardiog- 

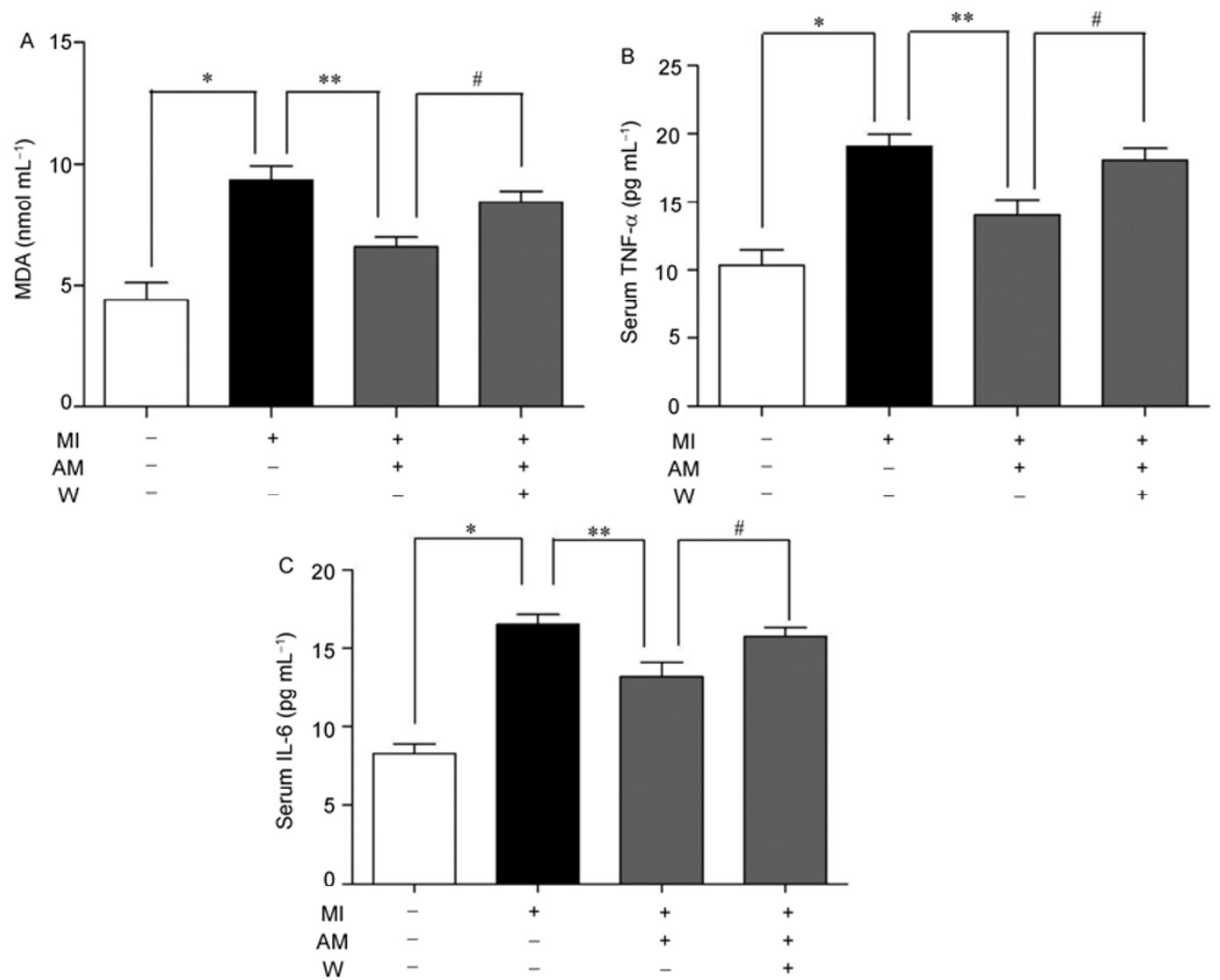

Figure 4 AM1241 decreased serum levels of LDH, TNF- $\alpha$ and IL-6 levels. A-C, Serum levels of LDH, TNF- $\alpha$ and IL-6 were measured by ELISA. *, $P<0.05$ vs. MI group; $\#, P<0.05$ vs. MI+AM group.
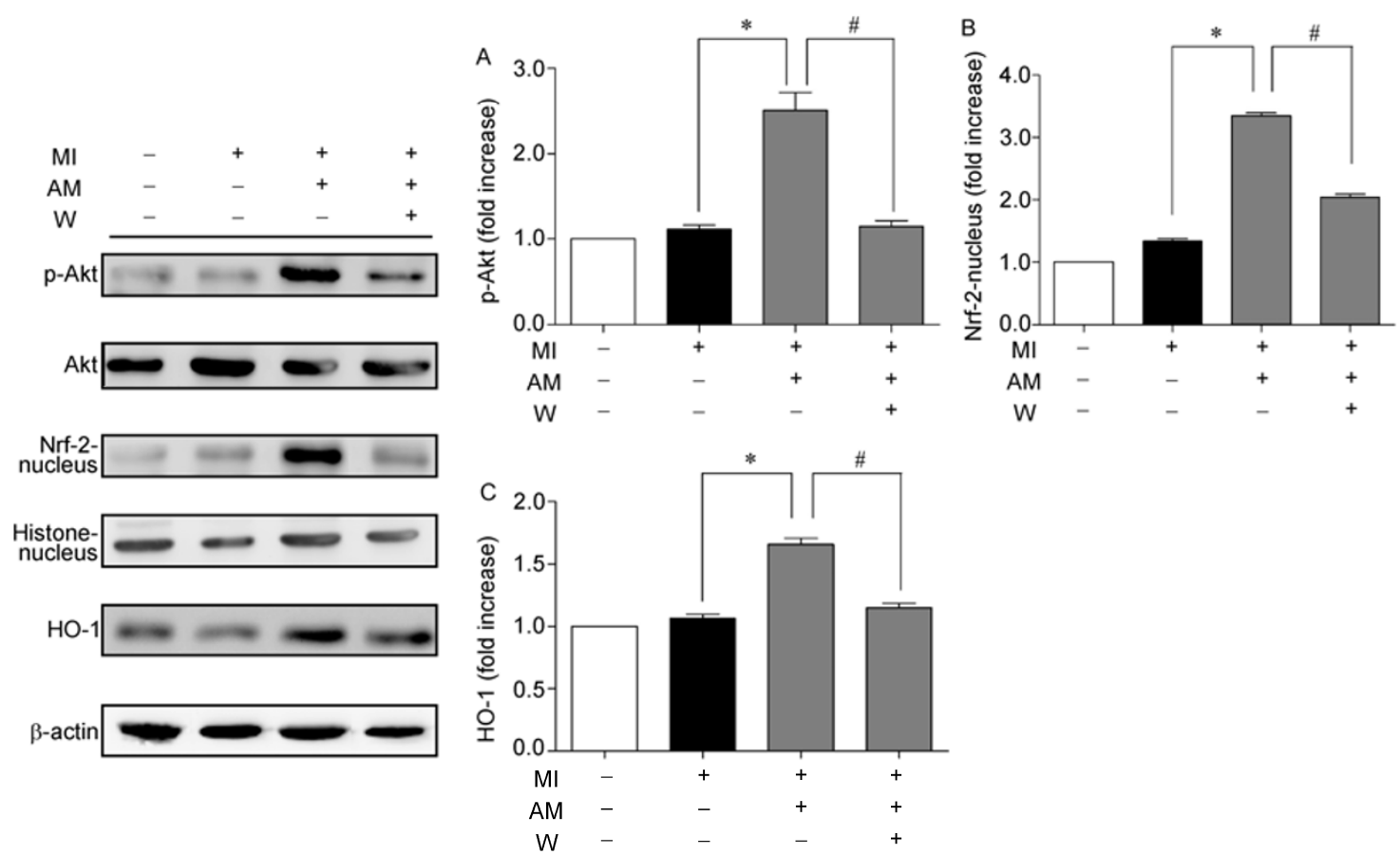

Figure 5 The protein expression of phosphorylation of Akt (p-Akt), Nrf2, HO-1 in myocardium was determined by Western blotting. Protein expression was quantified by the relative optical density ratio of p-Akt (A), Nrf-2 (B) and HO-1 (C). *, $P<0.05$ vs. MI group; \#, $P<0.05$ vs. MI+AM group. 


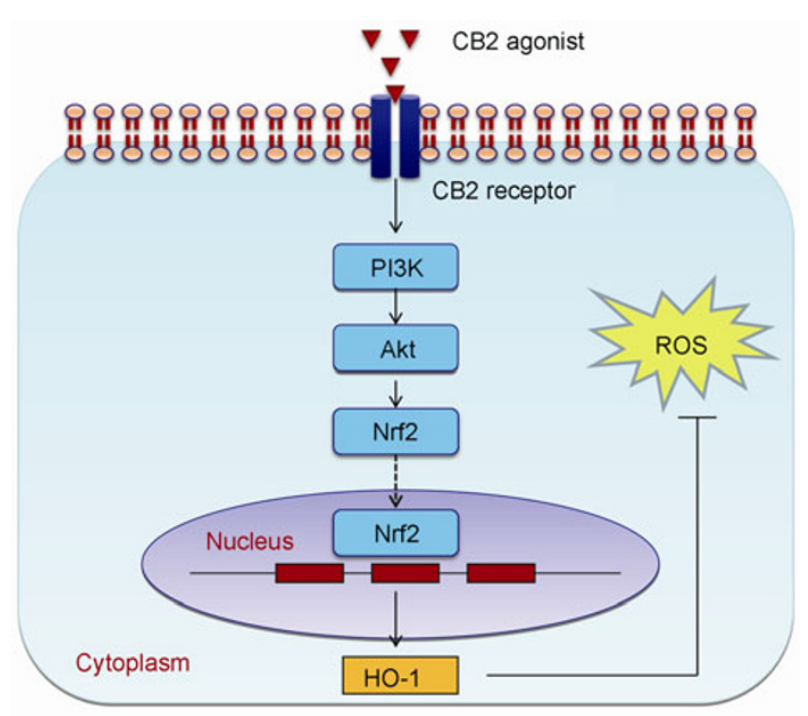

Figure 6 Proposed scheme for the mechanisms of anti-oxidative stress by CB2 agonist AM1241.

raphy and histology data demonstrated that AM1241 could significantly improve cardiac function. Furthermore, it also reduced fibrosis and prevented infarcted myocardium remodeling. These results were consistent with other previous reports [7]. With regard to precise mechanisms of cardiac protective roles of the $\mathrm{CB} 2$ receptor, some studies attributed them to anti-inflammatory and antioxidant properties. Nevertheless, the mechanisms by which $\mathrm{CB} 2$ receptor activation promotes cardiac repair and functional recovery through anti-inflammatory and antioxidant properties remain unclear.

Theoretically, it is somewhat controversial whether endogenous cardiac regeneration is the best route to cardiac repair to replace lost cardiomyocytes after MI [15]. Resident cardiac progenitor or stem cells are the major sources giving rise to regenerated cardiomyocytes [5]. However, the isolation, expansion and transplantation of CPCs are slow, expensive and low-quality, which inhibits their application in treating chronic heart failure after MI [16]. Therefore, it is necessary to provide new strategies to activate CPCs with cardiac regenerative potential in situ by drug, cytokine, or growth factor intervention [2]. Interestingly, the CB2 receptor activation was shown to promote activation and proliferation of some types of endogenous stem cells (e.g., hematopoietic stem/progenitor cells and neural progenitor cells) in vivo $[8,9]$. However, it is still unknown whether the CB2 receptor sensitization can activate endogenous cardiac progenitor cells in situ, which needs to be further investigated.

Our study demonstrated that AM1241 significantly raised expression of c-kit and Runx1 (typical progenitor cell markers) in the infarcted heart on POD 7. These results suggested that the $\mathrm{CB} 2$ receptor activation could accelerate resident CPC proliferation in vivo. Additionally, it has been demonstrated that the resident $\mathrm{CPC}$ activation is able to promote myocyte self-renewal and heart-tissue repair after MI [15]. In the present study, we immunostained heart tissue sections with cell proliferation marker ki-67 and found that the AM1241 administration increased ki67 expression in myocardium after MI, which indicated that selective CB2 agonist was capable of promoting cardiomyocyte regeneration and proliferation post-MI. Moreover, this increase of resident $\mathrm{CPC}$ activation and cardiomyocyte regeneration induced by selective CB2 agonist was probably related to the reduction of fibrotic area and enhancement in cardiac performance.

It was reported that CPCs that maintain homeostasis in a normal heart can be activated by pharmaceutical intervention, microRNA regulators or supporting cell stimulation after MI or heart failure to replenish injured or dead cardiomyocytes [2]. However, most of the CPCs may gradually decrease and be unable to play sustained roles, which becomes a major challenge to clinical application of myocardial regeneration therapy [17]. It was recently recognized that stem cell niches in vivo affected local CPC activation and function through inflammatory and growth cytokines secreted by cell neighbors [16]. Modulating the inflammatory response and oxidative stress in the infarcted myocardium to a moderate extent will provide a more suitable microenvironment for the CPC activation, proliferation and differentiation. In this study, we detected MDA, TNF- $\alpha$ and IL-6 levels in the infarcted heart with or without CB2 agonist administration, and observed that the CB2 agonist markedly decreased MDA, TNF- $\alpha$ and IL-6 levels in vivo. This indicated that CB2 receptor activation could inhibit inflammatory response and oxidative stress injury in myocardium, which improves cardiac post-ischemic microenvironment and benefits CPC activation and proliferation.

Next, it is necessary to investigate related mechanisms that may explain the anti-inflammation and anti-oxidant properties of the $\mathrm{CB} 2$ receptor activation. It is well known that $\mathrm{Nrf} 2$ is a key component for protecting against oxidative stress injury [18]. Under normal conditions, Keap1 combines with $\mathrm{Nrf} 2$ in the cytoplasm. Oxidative stress stimulus can disrupt the Nrf2/Keap1 complex and induce the translocation of Nrf2 into the nucleus. Nrf2 then binds to ARE (antioxidant-response element), which eventually results in increased expression of antioxidant genes such as heme oxygenase-1 (HO-1) and glutathione synthetic enzyme to resist oxidative stress injury [19]. Our Western blot results indicated that the $\mathrm{CB} 2$ receptor activation induced an increase of Akt phosphorylation, Nrf2 nuclear translocation and HO-1 expression, accompanied by cardiac function improvement, fibrosis decrease and CPC activation.

To further identify whether selective CB2 agonist plays cardiac protective roles via this signal cascade, we examined the effects of PI3K inhibitor Wortmannin on the infarcted heart in vivo after CB2 agonist administration. We found that Wortmannin cancelled the effects of the CB2 agonist including improving cardiac function enhancement, 
decreasing fibrotic area, reducing inflammatory cytokine secretion and oxidative stress. Additionally, the Wortmannin intervention also reversed up-regulation of c-kit, Runx1 and ki-67 expression caused by the CB2 receptor activation. These data illustrated that the treatment with PI3K inhibitor Wortmannin could inhibit the CB2 agonist-induced CPC activation and cardiomyocyte self-renewal after MI.

Finally, we observed that the Wortmannin treatment also suppressed Akt phosphorylation, Nrf2 nuclear translocation and HO-1 expression. Thus, the CB2 receptor activation promoted endogenous cardiac regeneration after $\mathrm{MI}$ at least partially via the PI3K/Akt/Nrf2 signaling pathway.

In conclusion, we illustrated for the first time that the CB2 receptor activation improved cardiac function and repair after MI through accelerating endogenous cardiac regeneration, which was related to ameliorating the cardiac post-ischemic microenvironment via modulating the PI3K/ Akt/Nrf2 signaling pathway.

This work was supported by the National Natural Science Foundation of China (81270168, 81090274, 81325009, 81090270 and F Cao BWS12J037), Innovation Team Development Grant by Ministry of Education of China (2010CXTD01, IRT1053), and the National Basic Research Program of China (2012CB518101).

1 Orlic D, Kajstura J, Chimenti S, Jakoniuk I, Anderson SM, Li B, Pickel J, McKay R, Nadal-Ginard B, Bodine DM, Leri A, Anversa P. Bone marrow cells regenerate infarcted myocardium. Nature, 2001, 410: 701-705

2 Wen Z, Mai Z, Zhang H, Chen Y, Geng D, Zhou S, Wang J. Local activation of cardiac stem cells for post-myocardial infarction cardiac repair. J Cell Mol Med, 2012, 16: 2549-2563

3 Malliaras K, Zhang Y, Seinfeld J, Galang G, Tseliou E, Cheng K, Sun B, Aminzadeh M, Marbán E. Cardiomyocyte proliferation and progenitor cell recruitment underlie therapeutic regeneration after myocardial infarction in the adult mouse heart. EMBO Mol Med, 2013, 5: 191-209

4 Oh H, Bradfute SB, Gallardo TD, Nakamura T, Gaussin V, Mishina Y, Pocius J, Michael LH, Behringer RR, Garry DJ, Entman ML, Schneider MD. Cardiac progenitor cells from adult myocardium: homing, differentiation, and fusion after infarction. Proc Natl Acad Sci USA, 2003, 100: 12313-12318

5 Garbern JC, Lee RT. Cardiac stem cell therapy and the promise of heart regeneration. Cell Stem Cell, 2013, 12: 689-698

6 Hajrasouliha AR, Tavakoli S, Ghasemi M, Jabehdar-Maralani P, Sadeghipour H, Ebrahimi F, Dehpour AR. Endogenous cannabinoids contribute to remote ischemic preconditioning via cannabinoid CB2 receptors in the rat heart. Eur J Pharmacol, 2008, 579: 246-252
7 Montecucco F, Lenglet S, Braunersreuther V, Burger F, Pelli G, Bertolotto M, Mach F, Steffens S. $\mathrm{CB}_{2}$ cannabinoid receptor activation is cardioprotective in a mouse model of ischemia/ reperfusion. J Mol Cell Cardiol, 2009, 46: 612-620

8 Palazuelos J, Ortega Z, Diaz-Alonso J, Guzman M, Galve-Roperh I. CB2 cannabinoid receptors promote neural progenitor cell proliferation via mTORC1 signaling. J Biol Chem, 2012, 287: 1198-1209

9 [Jiang S, Alberich-Jorda M, Zagozdzon R, Parmar K, Fu Y, Mauch P, Banu N, Makriyannis A, Tenen DG, Avraham S, Groopman JE, Avraham HK. Cannabinoid receptor 2 and its agonists mediate hematopoiesis and hematopoietic stem and progenitor cell mobilization. Blood, 2011, 117: 827-838

10 Gao E, Lei YH, Shang X, Huang ZM, Zuo L, Boucher M, Fan Q, Chuprun JK, Ma XL, Koch WJ. A novel and efficient model of coronary artery ligation and myocardial infarction in the mouse. Circ Res, 2010, 107: 1445-1453

11 Duerr GD, Heinemann JC, Dunkel S, Zimmer A, Lutz B, Lerner R, Roell W, Mellert F, Probst C, Esmailzadeh B, Welz A, Dewald O. Myocardial hypertrophy is associated with inflammation and activation of endocannabinoid system in patients with aortic valve stenosis. Life Sci, 2013, 92: 976-983

12 Li Q, Shi M, Li B. Anandamide enhances expression of heat shock protein 72 to protect against ischemia-reperfusion injury in rat heart. J Physiol Sci JPS, 2013, 63: 47-53

13 Barana A, Amorós I, Caballero R, Gómez R, Osuna L, Lillo MP, Blázquez C, Guzmán M, Delpón E, Tamargo J. Endocannabinoids and cannabinoid analogues block cardiac hKv1.5 channels in a cannabinoid receptor-independent manner. Cardiovasc Res, 2010, 85: 56-67

14 Hiley CR. Endocannabinoids and the heart. J Cardiovasc Pharmacol, 2009, 53: 267-276

15 Waring CD, Vicinanza C, Papalamprou A, Smith AJ, Purushothaman S, Goldspink DF, Nadal-Ginard B, Torella D, Ellison GM. The adult heart responds to increased workload with physiologic hypertrophy, cardiac stem cell activation, and new myocyte formation. Eur Heart J, 2012, Epub ahead of print

16 Ellison GM, Torella D, Dellegrottaglie S, Perez-Martinez C, Perez de Prado A, Vicinanza C, Purushothaman S, Galuppo V, Iaconetti C, Waring CD, Smith A, Torella M, Cuellas Ramon C, Gonzalo-Orden JM, Agosti V, Indolfi C, Galiñanes M, Fernandez-Vazquez F, Nadal-Ginard B. Endogenous cardiac stem cell activation by insulin-like growth factor-1/hepatocyte growth factor intracoronary injection fosters survival and regeneration of the infarcted pig heart. $\mathrm{J}$ Am College Cardiol, 2011, 58: 977-986

17 Di Nardo P, Forte G, Ahluwalia A, Minieri M. Cardiac progenitor cells: potency and control. J Cell Physiol, 2010, 224: 590-600

18 Anedda A, Lopez-Bernardo E, Acosta-Iborra B, Saadeh Suleiman M, Landazuri MO, Cadenas S. The transcription factor Nrf2 promotes survival by enhancing the expression of uncoupling protein 3 under conditions of oxidative stress. Free Rad Biol Med, 2013, 61C: 395-407

19 Dreger H, Westphal K, Wilck N, Baumann G, Stangl V, Stangl K, Meiners S. Protection of vascular cells from oxidative stress by proteasome inhibition depends on Nrf2. Cardiovasc Res, 2010, 85: $395-403$

Open Access This article is distributed under the terms of the Creative Commons Attribution License which permits any use, distribution, and reproduction in any medium, provided the original author(s) and source are credited. 